\title{
Numerical Simulation and Modelling of a Morphing Supercritical Airfoil in a Transonic Flow at High Reynolds Numbers
}

\author{
J.-B. TÔ ${ }^{1}$, D.M. ZILLI ${ }^{1}$, N. SIMIRIOTIS ${ }^{1}$, I. ASPROULIAS $^{1}$,D. SZUBERT $^{1}$ \\ A. MAROUF ${ }^{2}$, Y. HOARAU ${ }^{2}$, M. BRAZA $^{1}$ \\ ${ }^{1}$ Institut de Mécanique des Fluides de Toulouse, Unité Mixte C.N.R.S.-I.N.P.T. 5502, \\ Av. du Prof. Camille Soula, 31400 Toulouse, France \\ ${ }^{2}$ ICUBE, Unité Mixte C.N.R.S.-Université de Strasbourg 7357 \\ Bd. Sébastien Brant, 67400 Illkirch-Graffenstaden, France
}

\begin{abstract}
.
The flow dynamics and their morphing modification concerning the transonic flow around an Airbus A320 airfoil have been investigated via 2D simulations at a high Reynolds number. A distinctive flow topology, organised and chaotic occurs in this regime driven by appearance of coherent structures such as the VonKármán instability as well as the Kelvin-Helmholtz instability. When the Mach number and angle of attack both belong to a certain range of values, the shock wave develops a low-frequency motion along a specific distance on the suction side, issued from the development of transonic buffet instability. This phenomenon is crucial for the design because it leads to a high rise of drag and can trigger in extreme conditions dangerous dip-flutter modes. Electroactive morphing of the trailing edge region achieved by optimal vibration of piezo-actuators has proved capable to create vortex breakdown of the coherent structures and to act through an eddy-blocking mechanism to a considerable thinning of the shear layers and of the wake as has been proven in subsonic regime as shown by Scheller et al (2015). The eddy-blocking effect in the transonic regime has been studied in the present article in cruise-speed the conditions, following the studies by Szubert et al (2015) and Hunt, Eames, and Westerwee (2008). Accordingly, computations have been made to determine which type of actuation offers the best performance in terms of buffet dampening and aerodynamic efficiency as a whole. Lastly, it is shown that a flapping motion of the trailing edge can lock-in the frequency of the buffet phenomenon at the flapping frequency, which has potentially useful applications in terms of controlling and reducing shock oscillations.
\end{abstract}

Key words: instability, buffet, OES, supercritical wings, compressible flow.

\section{Introduction}

A considerable number of experimental and numerical studies have been devoted to the appearance and growth of buffet on a wing, i.e. the unsteady periodic motion of the shock over a transonic wing under specific flight conditions. Pioneering research driven by Marvin, Levy Jr., and Seegmiller (1980), Jacquin et al. (2009), Smits and Dussauge (2005) and Sajben and Kroutil (1981) have given a clear insight of the transonic flows with buffet phenomenon over wings.

Another study by Grossi, Szubert, et al. (2012) showed that the introduction of a splitter-plate at the trailing edge suppresses the Von Kármán mode, hence attenuating buffet. A strong interaction was thus identified between wake instabilities 
and the low frequency shock motion. While the trailing edge dynamics at the origin of buffet was relatively obscure up until then, an acoustic feedback mechanism has been found to be the source of buffet, which will be described hereinafter.

There is a strong correlation between the movement of the shock and the movement of the boundary layer, and this paper focuses on bringing to light additional insight on the mechanisms of buffet reduction thanks to hybrid morphing, while it is also shown that an appropriate trailing edge actuation can also present beneficial effects on the mean lift to drag ratio.

\section{Description of the numerical experiment}

\section{Geometry in use and flow parameters}

While transonic buffet realistically appears at high Reynolds numbers, at an order of magnitude of $10^{7}$ in cruising flight conditions, it was preferred to investigate buffet inside a numerical wind tunnel so that the computational mesh would be sufficiently small due to the strenuous refinement requirements that appear at very large Reynolds numbers.

The configuration used in this study is directly inspired from the wind tunnel experiments of the European project SMS - Samrt Morphing and Sensing for aeronautical configurations, www.smartwing.org/SMS/EU carried out by IMP-PAN Academy of Science in Gdansk, Poland. The chord of the prototype is of $0.15 \mathrm{~m}$. Buffet is thus investigated by means of a two-dimensional CFD computation as a fist step on the AIRBUS - A320 supercritical airfoil in reduced (laboratory) scale according to the ongoing experiments in free-stream Mach number of 0.78 and a chord-based Reynolds number of $2.06 \mathrm{M}$. The critical angle of attack $\alpha$ concerning the onset of buffet will be the objective of a detailed study concenring this configuraiton in 3D and by respecting the geometry of the upper and lower walls of the wind tunnel. In the present case, the objective is to study the morphing effect on the flow dynamics concerning a non confined 2D configuration as a first step enabling the design of the mmorphing prototype. In this case, onset of buffet has been observed in our studies at $\alpha=1.8^{\circ}$. At this angle of attack and flow parameters, the buffet frequency was found to be around $111 \mathrm{~Hz}$.

Another configuration at a higher angle of attack $\alpha=5^{\circ}$ is also used to enhance the buffet as well as the shock wave boundary layer interactions (SWBLI) that take place around the airfoil and within the turbulent wake. For this value of $\alpha$, a tangible separation is formed at the shock foot and the extent of the shock motion is broader than is the case for $\alpha=1.8^{\circ}$.

\section{Turbulence modelling and numerical parameters}

URANS modelling approaches as well as adaptive and more elaborate methods sensitizing the coherent structures development, as the OES - organised Eddy Simulation approach have been employed Braza et al. (2006) and Bourguet et al. (2008), Szubert et al. (2015). Furthermore, a $k-\omega$ Scale-Adaptive Simulation (SAS) approach was used as a first step until it was observed that the behaviour of the computed flow was non-physical, leading to a, excessive suction and flow detachment. 
A two-dimensional multi-block structured grid was used for the purpose of the computations.

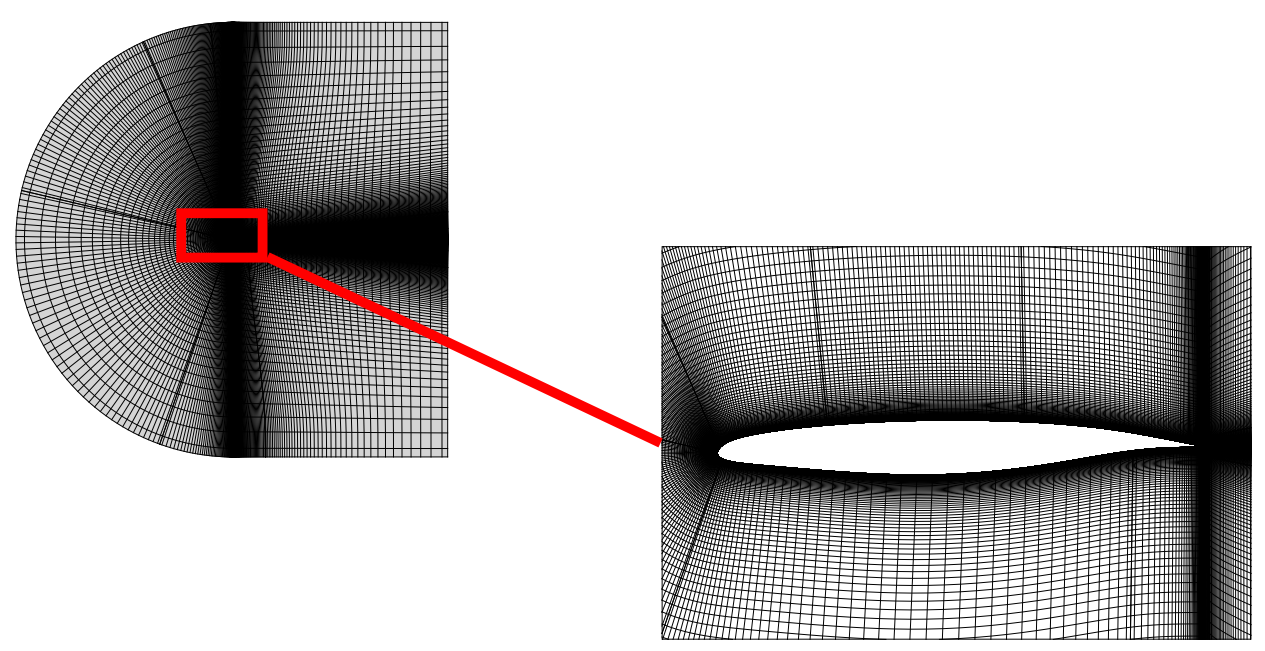

Two different grids have been examined and an exhaustive study of the grid influence, and time step has been carried out. The finally retained grid contains 160000 computational cells, and presents a C-H topology. A 190000-element mesh was generated afterwards with more elements in the wake region and on the suction side in order to better capture the shock and avoid dissipating wake vortices. The $y^{+}$ coordinate is of the order of $10^{-1}$ in the immediate vicinity of the airfoil walls.

\section{Numerical method}

The computations have been made by using the Navier-Stokes Multi Block (NSMB) code, thanks to the partnership of our research group within the NSMB European Consortium. Structured meshes are used and the Navier-Stokes equations for compressible flows is carried out following a finite-volume formulation. For this study, the temporal integration and spatial discretization schemes are chosen similar to the study from Grossi, M. Braza, and Hoarau (2014); the convective fluxes are discretized following a third-order Roe upwind scheme and time integration is performed through a dual-time stepping, second-order backward-difference scheme. The number of inner iterations was appropriate to ensure a sufficient convergence rate in respect of the flow requirements.

For this particular grid and geometry, it was determined that a time step $\Delta t_{s}=$ $5 \times 10^{-6} \mathrm{~s}$ was best suited as a compromise between speed and accuracy, as the time series for the force coefficients sensibly displayed the same results for $\Delta t_{s}$ and smaller time steps. An example is showcased in figure(1) where the lift coefficient $C_{l}$ for the $\alpha=1.8^{\circ}$, static wing at $\Delta t_{s}$ is shown to be identical to a $C_{l}$ computed for $\Delta t=10^{-6} \mathrm{~s}$.

\section{Results}

The buffet phenomenon associated with periodic shock motion and subsequent growth and thinning of the separated area are illustrated in the following figures. 

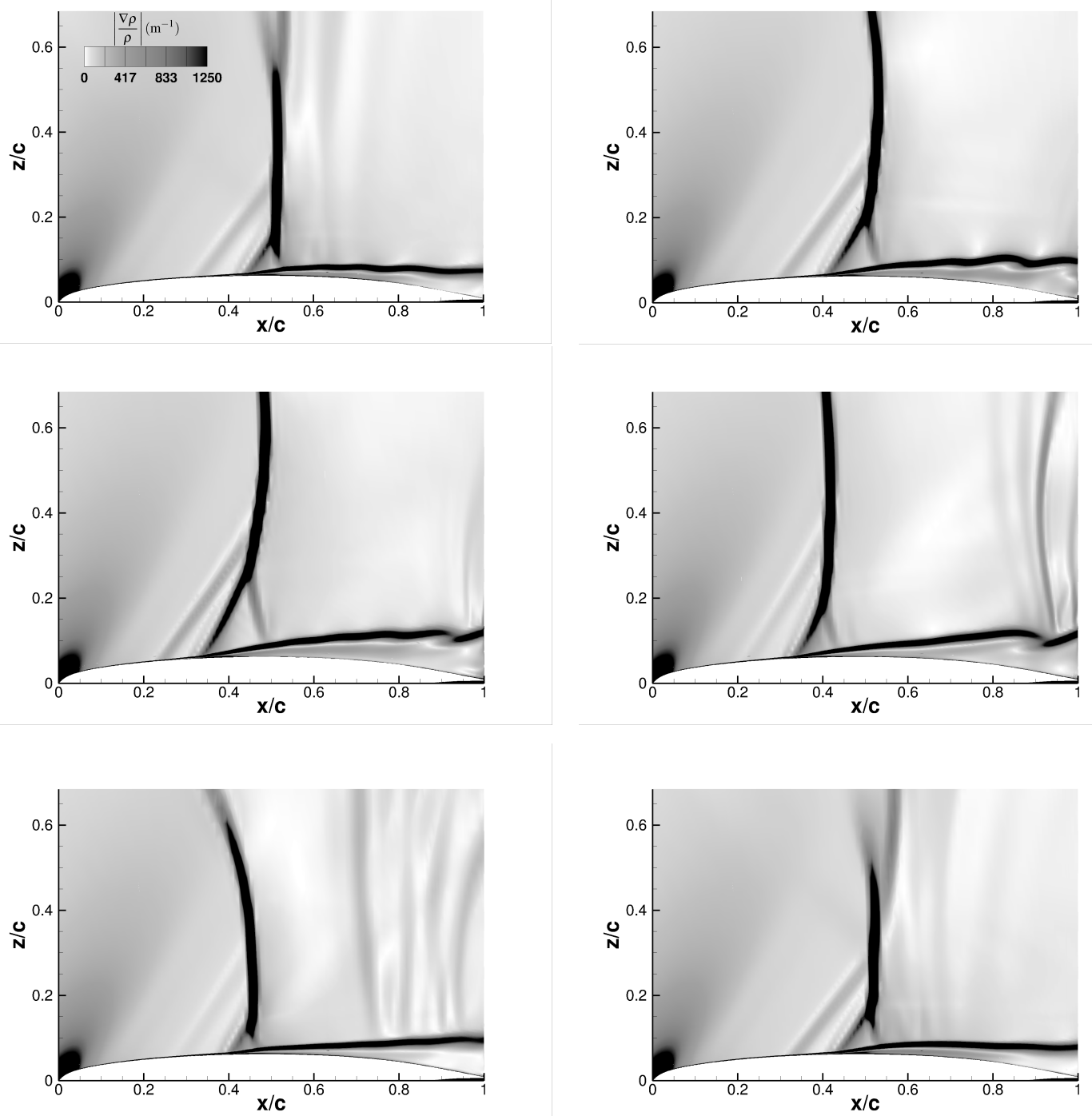

Figure 1: One buffet period represented by 6 snapshots. From left to right, top to bottom, the pictures correspond to times $t^{*}=\left\{0 ; \frac{1}{5} ; \frac{2}{5} ; \frac{3}{5} ; \frac{4}{5} ; 1\right\}$ where $t^{*}$ is a non-dimensional time defined by $t^{*}=\frac{t-t_{o}}{T_{b}}, T_{b}$ being the buffet period and $t_{0}$ the arbitrary moment chosen as the beginning of one buffet period. Here, it is chosen to be one of the moments when the shock is weakest, i.e. when it reaches its most downstream position (see Tijdeman (1977)).

An important issue is the appearance of the Von Kármán instability arising in the wake due to the presence of two inflection points in the velocity field, that is when $\partial_{z}^{2} u=0$. This instability then merges with the separated boundary layer and these two systems interact with one another in a viscous «zone»: The thickening of the boundary layer correlates with the shedding of Von Kármán vortices. In figure 2a it can be observed that when a coherent Von Kármán vortex is being released in the wake, where major viscous dissipation mechanisms occur, bumps appear alternatively in this viscous zone which act locally as a wedge to a compressible, transonic flow. The inviscid flow over this region is thus compressed again and gives birth to acoustic waves. These weak shocks appear intermittently whenever a vortex is 
(a)

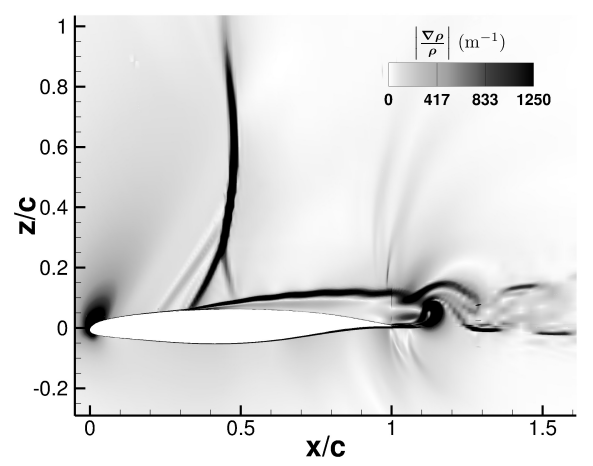

(b)

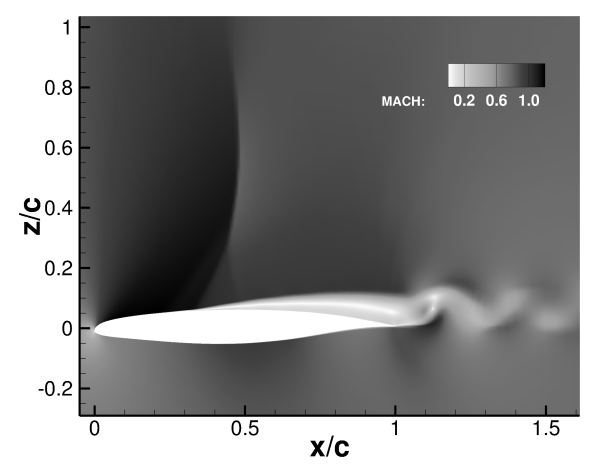

(c)

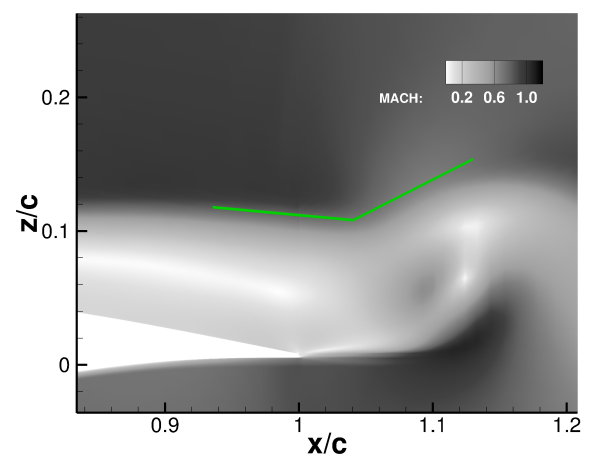

Figure 2: (a) A density gradient snapshot at $t^{*}=$

shed in the wake, both over the suction side of the wing and along the pressure side.Therefore, the von Kármán mode together with the shear-layer instability developed in the separated shear layers significantly affects by feedback mechanisms the SWBLI region and as seen in the next secion, is able to highly affect the buffet phenomenon. Indeed, the shear layers and the von Karman vortices near the trailing edge constitute a specific form of an effective obstacle containing the viscous effects and being thicker than the nominal airofil's configuration. The inertia effects associated with the compressibility around this virtual obstacle are the reason of formation of acoustic waves travelling upstream and modifying the SWBLI region, as well as the boundary layer upstream of the SWBLI. By means of the morphing, this study shows how optimally this feedback can operate.

These weak shocks - also known as Kutta waves - have a characteristically curved and almost circular shape (see schematic diagram 3), which comes from the diffraction they undergo when interacting with a vortex as mentioned in Gnani et al. (2014). These shocklets then grow and their wavefront propagates both upstream and downstream. Due to the transonic flow regime in the inviscid region over the separated boundary layer, the wave fronts decelerate while propagating upstream until they hit the lambda shock and impart their energy to it while fusing together. As it becomes stronger, the shock separates the boundary layer sooner and sooner, moving upstream along the wing until the acceleration pocket upstream the shock becomes too lean to allow the flow to gain speed. The shock thus loses strength and goes back downstream. As a direct consequence of this loss of strength, the boundary 


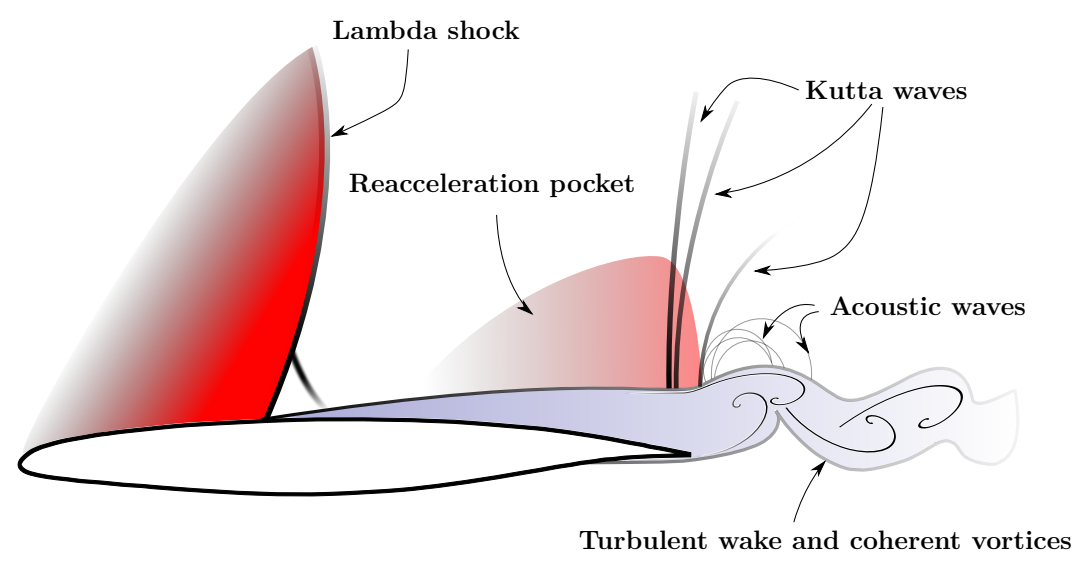

Figure 3: A schematic view of the main phenomena underlying buffet.

layer somewhat reattaches. Meanwhile, previously created Kutta waves are reaching the shock, which allows another cycle to begin.

\section{Force coefficients under the buffet effect}

The aforementioned physical mechanisms result in unsteady forces (fig. 1) characterized by a low-frequency periodic pattern repeating itself throughout the numerical experiment. Smaller oscillations also appear periodically in the grooves of the lift and drag signals, representing all the wake and boundary layer instabilities. By moving back and forth along the suction side, the shock thickens the boundary layer and induces a strong flow separation at its foot when it advances upstream, while the boundary layer becomes thinner when the shock moves downstream. If a strongly separated flow helps the Von Kármán and shear layer instabilities happen due to separation near the trailing edge, the reattachment that ensues causes them to disappear intermittently as can be seen on the .

In figure 4, the lift coefficient is plotted with respect to time in the $\alpha=1.8^{\circ}$ case without morphing. Although the unstable character of buffet is barely visible at this angle of attack if one were watching flow field snapshots, and even though separation is intermittent and weak, periodic patterns in the lift and drag coefficients as well as a distinct peak of energy in the Power Spectral Density (PSD) as seen in figure 7 are proofs that buffet still happens at that low angle of attack. When the trailing edge is slightly deflected upwards by an angle of $2^{\circ}$, the oscillations disappear, albeit at the expense of a reduction in performance, as the lift coefficient decreases on average. However, figure 5 shows that the drag coefficient also decreases by a substantial amount which means that on average, the lift to drag coefficient augments by $10 \%$. This can be explained by the fact that the slight upward deflection tends to reaccelerate the flow on the suction side, and the boundary layer will thus be sucked towards the surface of the wing, disfavouring both separation and the creation of wake instabilities. In turn, weakened instabilities mean weaker Kutta waves, and 


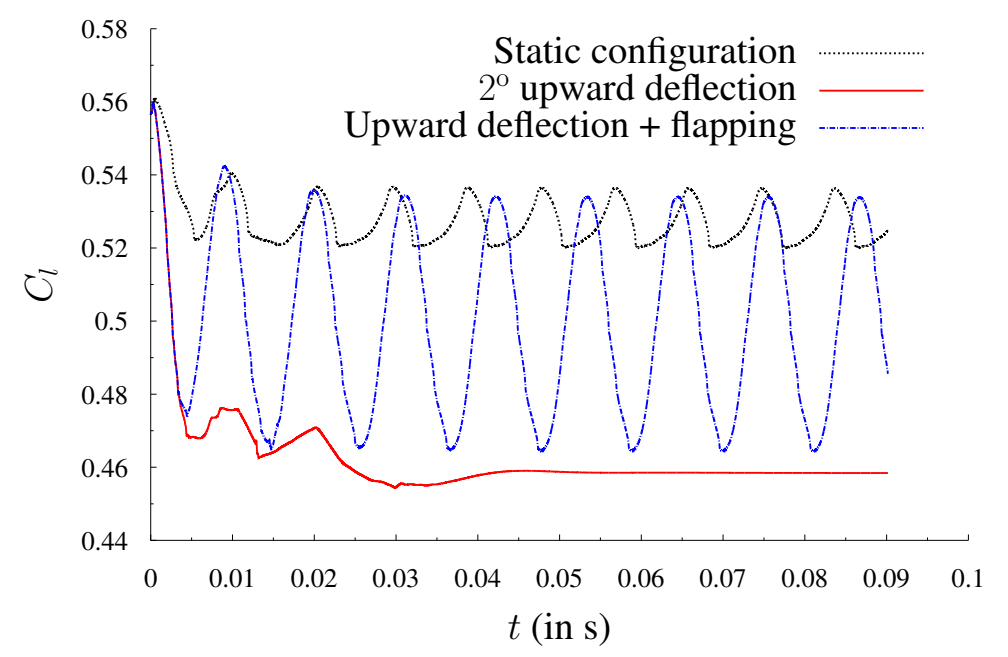

Figure 4: Lift coefficients for $\alpha=1.8^{\circ}$

a very faint interaction between said waves and the shock until buffet dies out altogether.

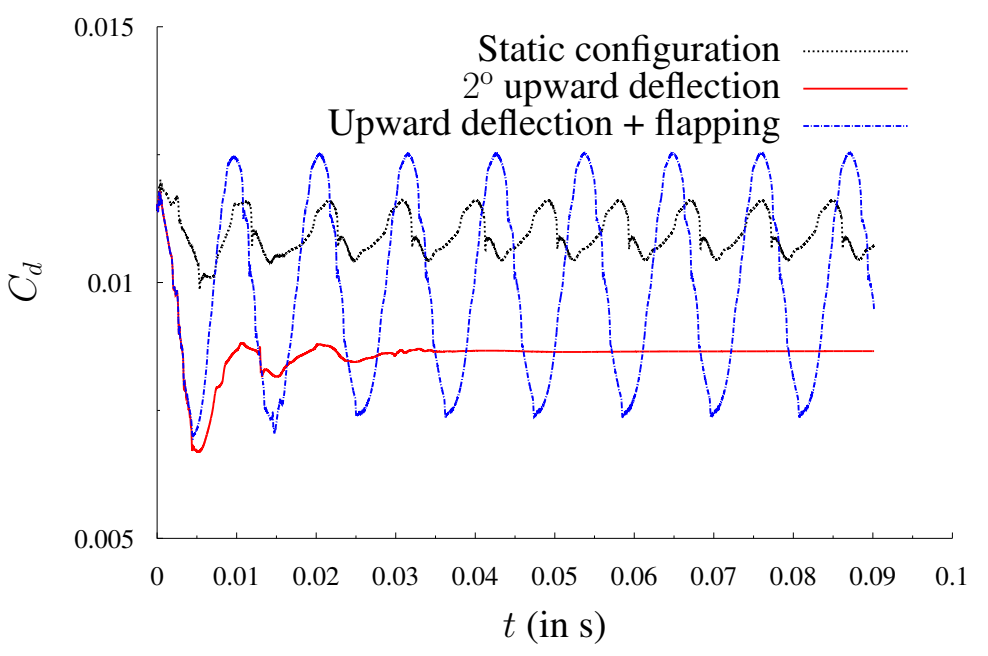

Figure 5: Drag coefficients for $\alpha=1.8^{\circ}$

This is less obvious for $\alpha=5^{\circ}$ because at this sharper incidence, separation is already too well-established to be suppressed by such a minimal deformation of the trailing edge. Figure 6 shows less of a difference between the static configuration and the morphing ones, although it is clear that morphing manages to reduce both lift and drag on average and to increase the mean lift to drag ratio, even at this angle of attack.

These results, computed when each signal is stationary, are summed up in table 1 . As was already pointed out, the improvements in terms of $\left\langle C_{l} / C_{d}\right\rangle$ are more prominent in the $\alpha=1.8^{\circ}$ case, because the angle of attack is reduced and separation does not occur as strongly as in the $\alpha=5^{\circ}$ case. Aa slight improvement can be noticed in all these configurations, with a notable increase in $\left\langle C_{l} / C_{d}\right\rangle$ for the small trailing edge deflection when the incidence is $1.8^{\circ}$.

While the force coefficients RMS $C_{f, \mathrm{rms}}=\left(\frac{1}{N} \sum_{i \in N \text { samples }}\left(C_{f}\left(t_{i}\right)-C_{f, \text { mean }}\right)^{2}\right)^{1 / 2}$ 

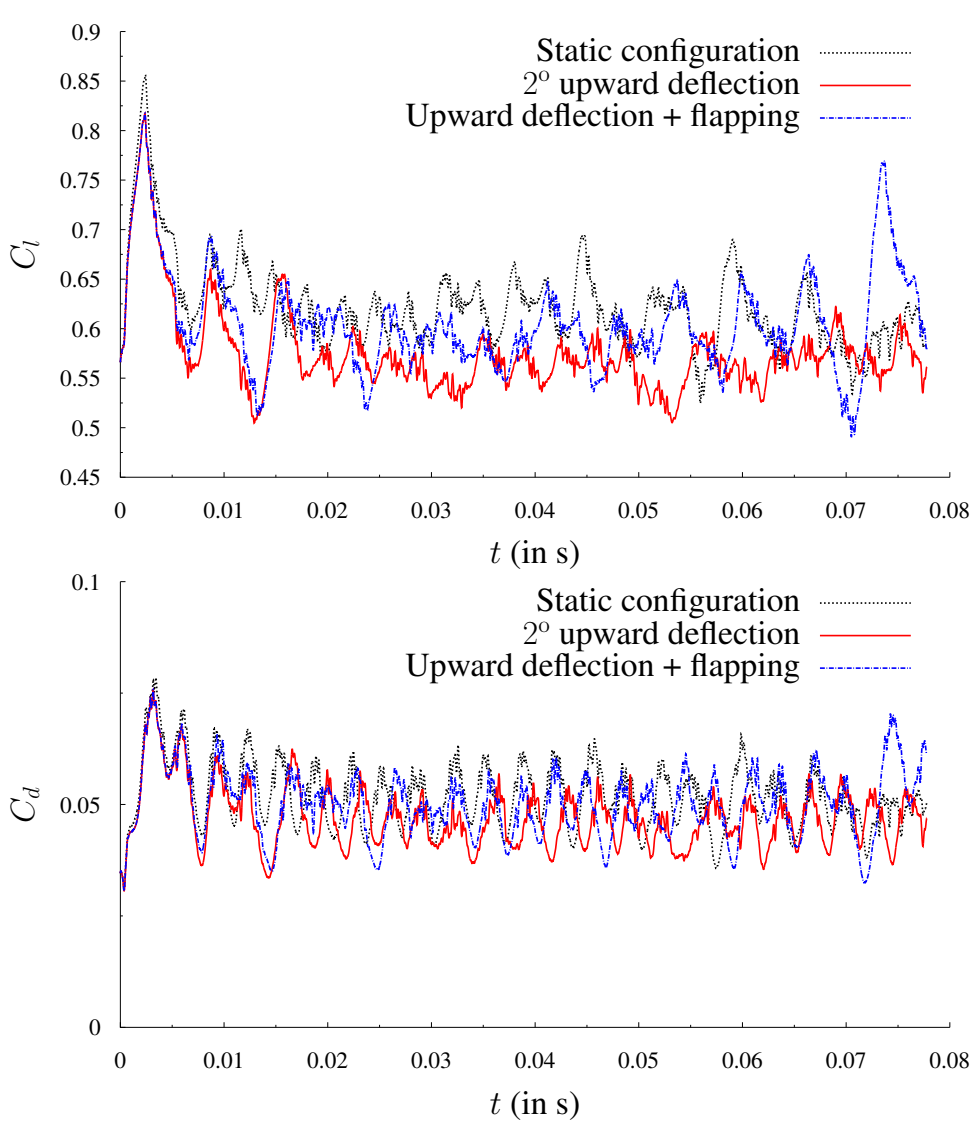

Figure 6: Force coefficients for $\alpha=5^{\circ}$.

\begin{tabular}{|c|c|c|c|c|}
\hline Incidence & \multicolumn{2}{|c|}{ Angle of attack 1.8 } & \multicolumn{2}{|c|}{ Angle of attack 5 } \\
\hline Type of actuation & $\mathrm{D}$ & $\mathrm{D}+\mathrm{F}$ & $\mathrm{D}$ & $\mathrm{D}+\mathrm{F}$ \\
\hline$\frac{\left\langle C_{l} / C_{d}\right\rangle-\left\langle C_{l} / C_{d}\right\rangle_{\text {static }}}{\left\langle C_{l} / C_{d}\right\rangle_{\text {static }}} \times 100$ & $+10.4 \%$ & $+4.3 \%$ & $+2.5 \%$ & $+0.4 \%$ \\
\hline
\end{tabular}

Table 1: Relative mean lift over drag ratio gain compared to the static case. Note that D means " $2^{\circ}$ deflection" and " $\mathrm{D}+\mathrm{F}$ " is the superposition of an immobile upward deflection and a $90 \mathrm{~Hz}$ flapping motion.

shrink dramatically for $\alpha=1.8^{\circ}$ when a small trailing edge deflection is applied, this is less evident for $\alpha=5^{\circ}$. An important issue of the present study is that an upward $2^{\circ}$ deflection is still sufficient to reduce both lift and drag rms quite considerably. Most crucially, it can be seen on energy spectra that morphing essentially modifies buffet through a dampening of flow instabilities in the wake region: By modifying the high frequency region of the spectrum $(S t>5)$ and diminishing strength of all flow instabilities in this region, the non-linear interactions with the buffet expressed through the frequency peaks in between the buffet bump and Von Kármán mode as well as btween the von Kármán and the higher-frequency Kelvin-Helmholtz modes in the energy spectrum, are suppressed. This is the main mechanism of buffet annihilation.

In the energy spectrum of figure 7, it is noticeable that most of the modes have been dampened by the trailing edge deflection, while flapping modifies the behaviour of 


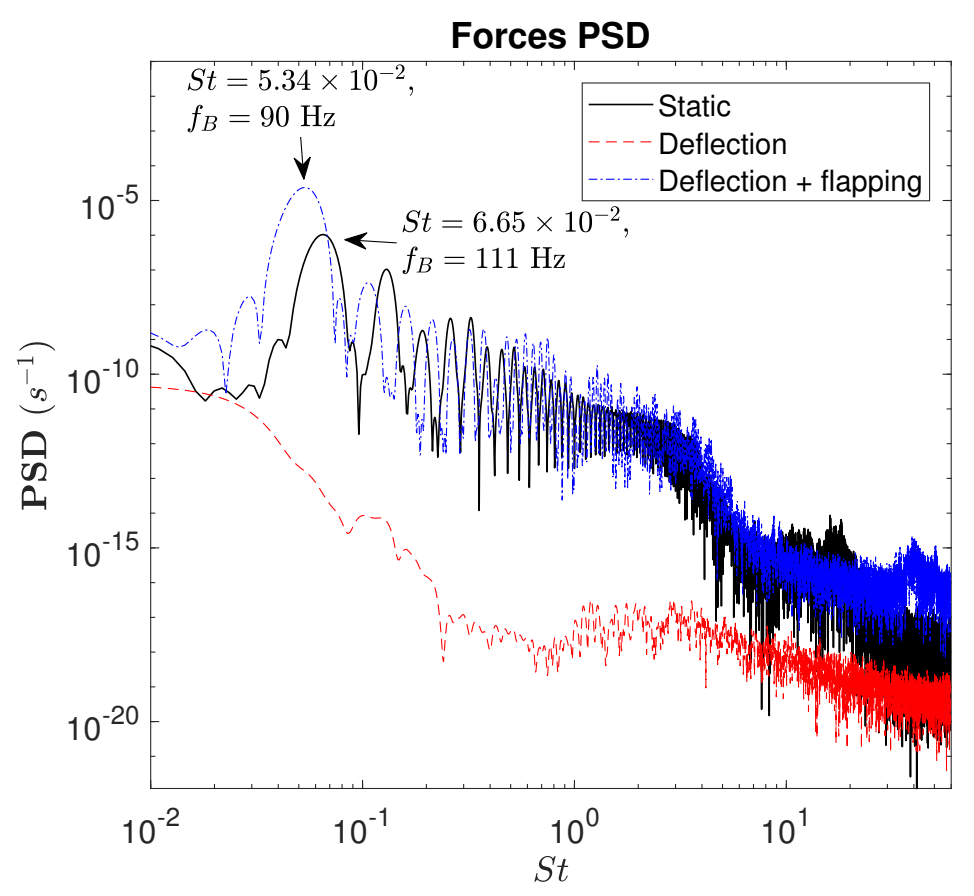

Figure 7: Energy spectra for $\alpha=1.8^{\circ}$.

high frequency phenomena all the way to the left where intermediate bumps (which are harmonics of the buffet mode) are shifted to the left. This effectively displaces the natural frequency of the buffet instability which now takes the exact same value as the frequency of actuation.

\section{A frequency lock-in}

A frequency lock-in is highlighted when the flapping frequency is modified. Flapping was performed at $f_{\text {act }}=70 ; 80 ; 90 ; 100$ and $120 \mathrm{~Hz}$, which corresponds to $S t=$ $4.16 \times 10^{-2} ; 4.75 \times 10^{-2} ; 5.34 \times 10^{-2} ; 5.94 \times 10^{-2}$ and $4.16 \times 10^{-2}$ respectively.

As is shown in figure 8 , the large bump that corresponds to buffet perfectly coincides with the actuation frequency, which shows the strong influence of morphing on the shock motion. This may also explain why it is relatively difficult to act upon buffet at a larger angle of attack. A PSD for $\alpha=5^{\circ}$ shows (cf. figure 9 ) that buffet does not occur at a single frequency, which means that it does not appear as one single peak or bump in the PSD, but as a clustering of multiple peaks.

This translates into a frequency modulation due to the intense non-linear interaction between wake instabilities and the shock, as can be seen in figure 10. The small upward deflection cannot influence this interaction strongly enough and the PSD remains pretty much the same, with a slight drop in energy for low frequency modes. However, even though flapping does not influence buffet as much as it did for $\alpha=$ $1.8^{\circ}$, this actuation excites a small bump that exactly corresponds to the morphing frequency, which indicates that a frequency lock-in still arises at that angle of attack (cf. figure 11). 

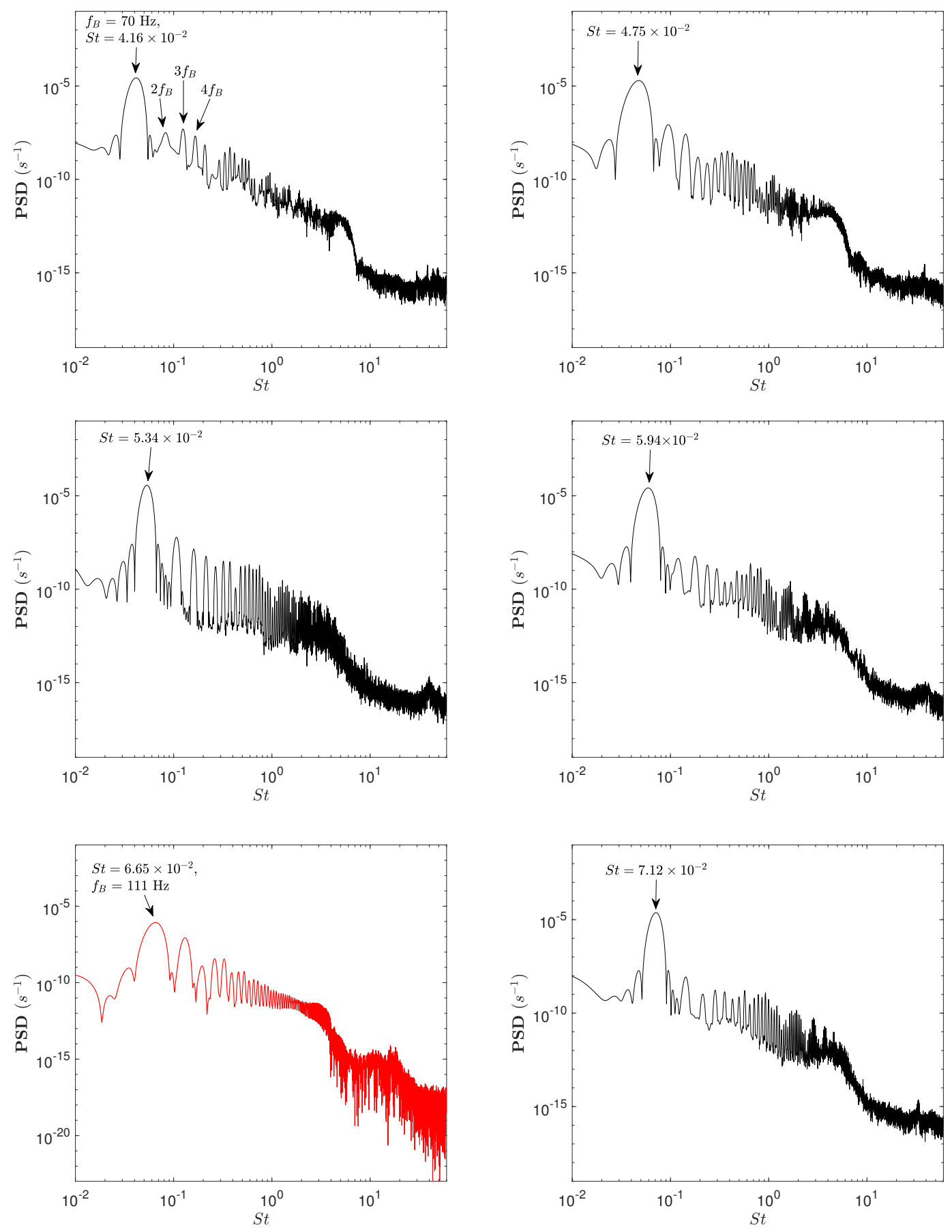

Figure 8: Power Spectral Density plots of the lift coefficient for different actuation frequencies. Black curves: PSD estimation via a Welch method for the following actuation frequencies: $f_{\text {act }}=\{70 ; 80 ; 90 ; 100 ; 120\}$ Hz. Red curve: Static configuration, i.e. unmorphed airfoil at an angle of attack $\alpha=1.8^{\circ}$.

\section{Conclusions}

The present study analyses the transonic buffet mechanisms related to coherent vortex formation in the separated shear layers and in the near wake, as well as their 


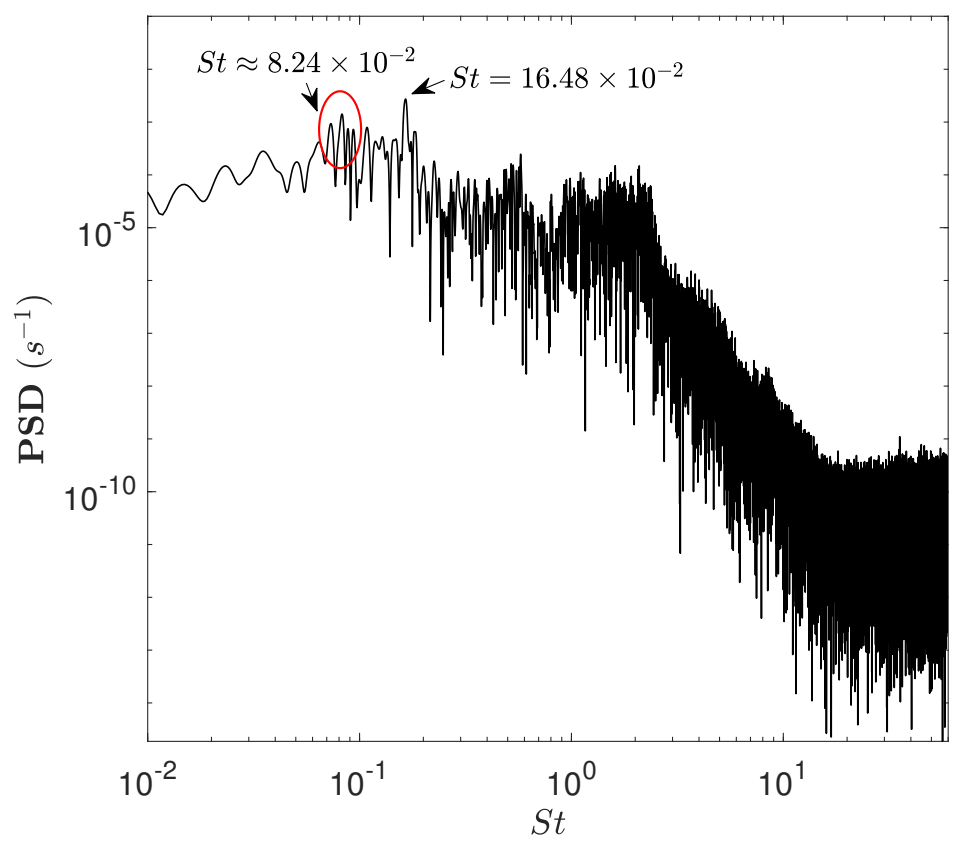

Figure 9: PSD for $\alpha=5^{\circ}$. A slightly predominant frequency stands out at $S t=16.48 \times 10^{-2}$, but it appears that a cluster of frequencies better characterizes buffet than just a single peak.

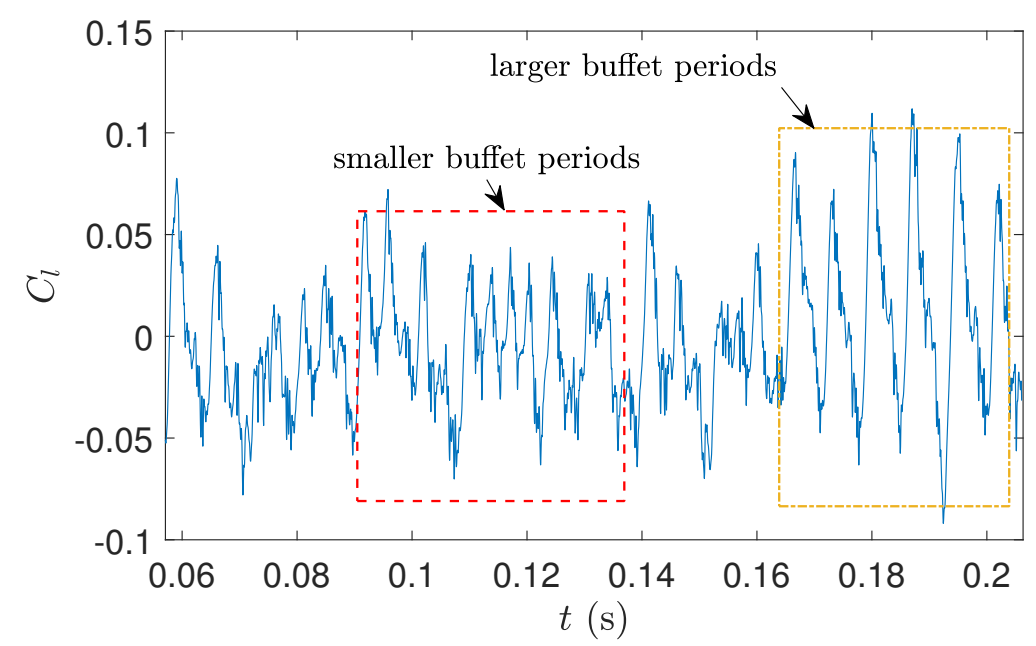

Figure 10: A frequency modulation can be observed, with zones corresponding to a low-frequency buffet, others to a higher-frequency phenomenon.

feedback effct towrds the SWBLI area.

2D computations by using the OES approach around a static and morphing A320 airfoil have shown that slightly deflecting and optimally actuating the trailing edge region in the transonic regime corresponding to the cruise phases of flight, is able to considerably manipulate the surrounding vortex structures and the shear layers and affect the buffet dynamics. A frequency lock-in of the buffet mode and the actuation freequency has been shown by specific slight deformations and vibrations of the near-trailing edge area. 


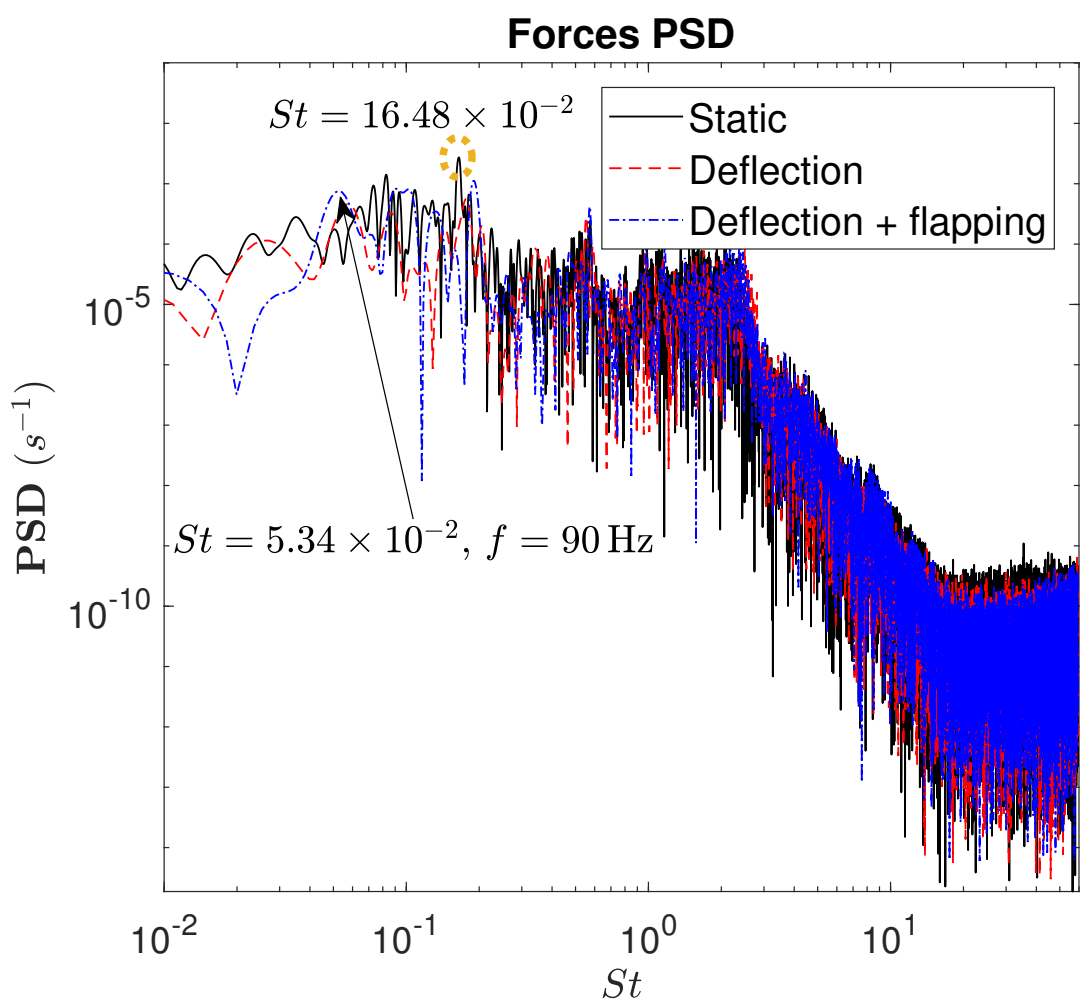

Figure 11: An energetic bump appears at $f=90 \mathrm{~Hz}$ for the deflection + flapping case. This is a testament of frequency lock-in at $\alpha=5^{\circ}$.

\section{Acknowledgements}

The authors are grateful to the LAPLACE Laboratory team of electroactive actuators and to the National Supercomputing centres CALMIP, CINES and IDRIS for the Computer allocation.

\section{Bibliography}

Bourguet, R. et al. (2008). "Anisotropic Organised Eddy Simulation for the prediction of nonequilibrium turbulent flows around bodies". In: Journal of Fluids and Structures 24(8), pp. 12401251. ISSN: 08899746. DOI: $10.1016 / \mathrm{j} \cdot$ jfluidstructs.2008.07.004

Braza, M et al. (2006). "Turbulence modelling improvement for highly detached unsteady aerodynamic flows by statistical and hybrid approaches". In: ECCOMAS CFD 2006 : European Conference on Computational Fluid Dynamics, September 5-8, 2006.

Gnani, F. et al. (2014). "Experimental investigation on shock wave diffraction over sharp and curved splitters". In: Acta Astronautica 99(1), pp. 143-152. ISSN: 00945765. DOI: 10.1016/j. actaastro.2014.02.018

Grossi, F., Braza, M., and Hoarau, Y. (2014). "Prediction of Transonic Buffet by Delayed DetachedEddy Simulation". In: AIAA Journal 52(10), pp. 2300-2312. ISSN: 0001-1452. DOI: 10.2514/ 1.J052873 URL: http://arc.aiaa.org/doi/10.2514/1.J052873.

Grossi, F., Szubert, D., et al. (2012). "Numerical simulation and turbulence modelling of the transonic buffet over a supercritical airfoil at high Reynolds number". In: Proceedings of the ETMM9 9th International ERCOFTAC Symposium on Engineering Turbulence Modelling and Measurements. Thessaloniki, Greece. 
Hunt, J.C.R., Eames, I., and Westerweel, J. (2008). "Vortical Interactions with Interfacial Shear Layers". In: IUTAM Symposium on Computational Physics and New Perspectives in Turbulence. Ed. by Yukio Kaneda. Springer Netherlands: Dordrecht, pp. 331-338. ISBN: 978-1-40206472-2.

Jacquin, L. et al. (2009). "Experimental Study of Shock Oscillation over a Transonic Supercritical Profile". In: AIAA Journal 47(9), pp. 1985-1994. ISSN: 0001-1452. DOI: $10.2514 / 1.30190$. URL: http://arc.aiaa.org/doi/10.2514/1.30190.

Marvin, J.G., Levy Jr., L.L., and Seegmiller, H.L. (1980). "Turbulence Modeling for Unsteady Transonic Flows". In: AIAA Journal 18(5), pp. 489-496. ISSN: 0001-1452. DOI: 10.2514/3. 50782. URL: https://arc.aiaa.org/doi/abs/10.2514/3.50782.

Sajben, M. and Kroutil, J.C. (1981). "Effects of Initial Boundary-Layer Thickness on Transonic Diffuser Flows". In: AIAA Journal 19(11), pp. 1386-1393. ISSN: 0001-1452. DOI: 10.2514/3. 60075 .

Scheller, J. et al. (2015). "Trailing-edge dynamics of a morphing NACA0012 aileron at high Reynolds number by high-speed PIV". In: Journal of Fluids and Structures 55, pp. 42-51. ISSN: 10958622. DOI: $10.1016 / \mathrm{j} \cdot \mathrm{jfluidstructs.2014.12.012.}$

Smits, A.J. and Dussauge, J.P. (2005). Turbulent Shear Layers in Supersonic Flow, p. 410. ISBN: 978-0-387-26305-2. URL: http : / / www . springer . com / physics / classical + continuum + physics/book/978-0-387-26140-9.

Szubert, D. et al. (2015). "Shock-vortex shear-layer interaction in the transonic flow around a supercritical airfoil at high Reynolds number in buffet conditions". In: Journal of Fluids and Structures 55, pp. 276-302. ISSN: 10958622. DOI: $10.1016 / \mathrm{j} \cdot \mathrm{jfluidstructs.2015.03.005.}$ URL: http://dx.doi.org/10.1016/j.jfluidstructs.2015.03.005.

Tijdeman, H. (1977). "Investigation of the transonic flow around oscillating airfoils". In: National Aerospace Lab. Amsterdam, Netherlands TR-77-090U. DOI: b07421b9-136d-494c-a161b188e5ba1d0d. 